\title{
Brain Tumor Diagnosis Using MR Image Processing
}

\author{
Sura Yarub Kamil, Mohammed Sahib Mahdi Altaei* \\ Computer Science Department, College of Science-Al-Nahrain University, Baghdad, Iraq
}

\begin{tabular}{ll}
\hline \multicolumn{1}{c}{ Articles Information } & \multicolumn{1}{c}{ Abstract } \\
\hline Received: & Magnetic Resonance Imaging (MRI) images of brain are a process of high \\
26.02 .2020 & importance to diagnosing brain tumors. Brain tumor is an abnormal growth of \\
Accepted: & cells in the brain. These tumors may be benign or malignant. The use of \\
14.05 .2020 & computer technologies became widely used to store and manage medical images \\
Published: & for supporting medical decision and improve the accuracy of radiologists with a \\
04.06 .2020 & reduction of time in the interpretation of images. The present study aimed to \\
& establish a Computer-Aided Detection and Diagnosis (CADD) system dealing \\
& with medical MRI for classifying input digital image into normal or abnormal \\
& tumors, also the type of abnormal case is diagnosed into benign or malignant \\
& tumor. The proposed method is considered to be contained four stages within, \\
& they are: Pre-processing stage, image segmentation for determining the Region \\
Keywords: & of Interest (ROI), Feature extraction based on Scale Invariant Feature \\
MRI images & Transform (SIFT) descriptor, and then classification. The results of classification \\
Brain tumors & are evaluated by cross validation technique, in which the dataset are divided \\
Detection & into training set and testing set. To evaluate the achieved results, the \\
Classification & classification is carried out using two levels for each case: logistics technique is \\
SIFT & used to check the results of normal case, and random forest to check the results \\
& of abnormal cases. Results of normal classification showed that the accuracy of \\
& applying Logistic Regression was 93.3\%, whereas the classification score of \\
& abnormal cases was 99.9\% for Random Forest, which ensure the success of the \\
& classification system and correct path of the computations.
\end{tabular}

DOI: $10.22401 /$ ANJS.23.2.10

*Corresponding author: msm@sc.nahrainuniv.edu.iq

\section{Introduction}

Tumor is an anomalous mass that may exist inside or on the brain. Two different terms are used for this anomalous and abnormal part in the brain, they are: Tumor and Cancer and it is very risky for human life [1]. There are different techniques to diagnosis the brain tumor like X-Ray, Computed Tomography, Positron Emission Tomography, MRI and others. MRI can be defined as a technique of imaging utilizing for visualizing the body's internal parts, MR images could be considered as a tool of high importance in early detection of brain tumors. Recently, MRI interpretation depends on radiologist's opinion [2]. The segmentation of images indicated the process used to partition digital images to multiple segments, each one of these segments have pixel's set. The pixels that are existing in region are comparable with regard to certain homogeneity standard like texture, intensity, or color [3]. Contour can be defined as a type related to the segmentation method which utilizes the forces of energy as well as the constraints to segregate pixels of interest from images for more analysis and processing. Contour defined as an active model which is used for segmentation and considered as boundaries that are used for area of interest needed in images [4]. SIFT is considered as new class of algorithms that emerged to be used for object recognition, the basic idea is the use of local features matching by transforming the content of image into coordinates of local features which then invariant for translation, rotation, and scaling [5]. This method suggested by David Lowe for extracting features which are distinctive and invariant from image for recognizing different images of same object [6]. Classification can be considered as the process used to classify input patterns in analogous classes. In the case when input dataset is specified through the class membership, it will be referred to as supervised learning. It uses two processing phases (training and testing). With regard to training phase, the properties related to the image's features will be isolated, distinctive definition regarding each one of the classification categories will be generated. With regard to the 


\title{
Al-Nahrain Journal of Science
}

\author{
ANJS, Vol.23 (2), June, 2020, pp. 67 - 74
}

testing phase, such feature space partitions will be utilized for classifying the features of image [7]. A major step in the process of classification is the adequate selection regarding the method of classification. Generally, the methods of classification could be classified into two types; unsupervised classification and supervised classification [8]. Recently, the major classifiers utilized for classification of images are: Logistic Regression (LR) and Random Forest (RF). Each classifier has benefits and drawbacks related to its applications. Generally, sample imaged in each one of the classes will be allocated to one class, comparison will be conducted each two classes and the sample will be allocated to higher votes related to each one of the classes [9].

\section{Related Works and Contribution}

There are many literatures are found in the field of MRI processing, some of them are concerned with medical applications for purpose of designing a computer aided system to diagnose human diseases. The important related works that discussing such interest point besides our contribution are mentioned in the following:

\subsection{Related Work}

Many published researches included the problem of medical image classification based on features extracting for obtaining detailed information that by which magnetic resonance images were classified to help the decision-making physician. Amulya and Prathibha in 2016 used two feature extraction methods: Scale Invariant Feature Transform (SIFT) and Speeded up Robust Features (SURF). SIFT approach is utilized for detecting images with large corners and after that extracting them. SURF is representing a speed technique for extracting features in comparison to SIFT.K-Nearest Neighbor KNN classifier has been utilized for classifying images depending on features which have been extracted from the two approaches. Thus, such combined processes have been utilized for classifying non-tumor and tumor images with more accuracy [10]. Alfonse et al. in 2016 developed a brain tumor diagnostic system. The system determines the tumor's type which is malignant or benign using MRI images. The system has been assessed according to series of brain tumor images. The experimental results demonstrate that the suggested system has a classification accuracy of $98.9 \%$. For the purpose of classifying input images as abnormal or normal, they applied SVM that is a systematic technique for two class problems. SVM needs 2 phases; training and testing. SVM could be trained through features provided as input to the learning algorithm. Throughout the training phase, SVM will find proper margins between 2 classes. Features will be labeled depending on the class related to certain class [11]. Gurusamy et al. in 2017 proposed a novel approach to detect and extract tumors on MRI images, these images will enable the physicians in studying and diagnosing tumors or diseases in brain. This study has the aim on enabling physicians and radiologists to have another opinion when diagnosing. MRI image obtained from machine will be subjected to analysis in the work. Major pre-processing will be achieved with the use of filters for removing noise. De-noised images are segmented, feature extractions will be achieved. Features will be extracted with the use of wavelet transform. Features will be provided to classifier that utilizes binary tree support vectors for classification. The process of classification is put to comparison with traditional approaches [12]. Bahadure et al. in 2017 examined Berkeley Wavelet Transformation (BWT) based brain tumor segmentation. The authors enhanced the quality rate and precision regarding SVM based classifier, where related features will be extracted from all segmented tissues. Experimental results related to the suggested approach were assessed and confirmed for quality and performance analysis on the magnetic resonance brain images. The experimental results accomplished (96.51\%) precision, (94.2\%) specificity, and (97.72\%) sensitivity, showing the efficiency related to the suggested approach to identify abnormal and normal tissues from the MR images of brain [13]. Bahadure, N. et al. in 2018 improved the performance related to detecting tumors; the authors examined comparative method for various techniques of segmentation and chose the optimum one through putting their segmentation score to comparison. Also, for improving the accuracy of classification accuracy, genetic algorithm has been used for tumor stage's automatic classification. . Experimental results related to the suggested approach have been assessed and verified for quality and performance analysis on the MRI images, depending on dice similarity index coefficient, sensitivity, accuracy, and specificity. Experimental results reached $92.36 \%$ sensitivity, $91.42 \%$ specificity, and $92.03 \%$ accuracy [14]. Dandu et al. in 2019 claimed detecting pancreatic tumors and brain tumors with the use of Decision Based Couple Window Median Filter (DBCWMF) algorithm, Statistical Region Merging (SRM), Cat 


\title{
Al-Nahrain Journal of Science
}

\author{
ANJS, Vol.23 (2), June, 2020, pp. 67 - 74
}

\begin{abstract}
Swarm Optimization and SIFT (CSO-SIFT) extraction and classification through Back Propagation Neural Network (BPNN) has been provided. DBCWMF work efficiently in preprocessing in comparison to Median, segmentation will be achieved with using SRM algorithm. After that, feature selection approaches SIFT and CSO are utilized to detect part in the tumor images that has been impacted and last classification via BPNN classification work effectively [15]. Polepaka, et al. in 2019 used Interactive Diagnosis Support System (IDSS) method and indicated the drawbacks regarding non-illumination and the low contrast that is related to MRI image of brain tumor which impact the process of precise classification regarding the image. Therefore, IDSS has been utilized in 3 stages which are image preprocessing to enhance the non-illuminated features, image classification and feature extraction that are achieved with the use of 2phase interactive SVM Classification. Local binary patterns in feature extraction for precise classification regarding unusual and usual MR images of brain [16].
\end{abstract}

\subsection{Contribution}

The present research aim to make the highly accurate SIFT technique identifies keys and important points in MRI images. Also, it is intended to improve the capabilities of the SIFT to be able to determine the type of tumor if it is tumors of meningitis or malignant tumor and also tumor degree or type, this is requires to build a system to analyze MRI images of the tumorbearing brain automatically, and use different classifiers to find the best classifier that showed compatibility with the used descriptor to obtain best classification results.

\section{The Proposed Classification System}

The concept of sequential stages based query processing and description has been used to model the proposed method. It is claimed that these stages can beneficially be combined to prepare a significant fast and efficient MRI classification achievement. The generic structure of the proposed brain tumor classification method is shown in figure 1. It is shown that the proposed method is established to be consisted of two phases: the training and classification. The training phase is responsible on collecting different classes found in sample images to be stored as comparable models of database. Whereas the classification phase is responsible on verifying the test MRI in comparison with the trained models found in the database. Both phases are composed of three stages include: image preprocessing, image segmentation, and image description that related to features extraction. Features extraction attempts to estimate the SIFT key points for just ROI of the test image. Last stage is a comparison based on descriptive features between query image and training classes found in the database, the result of the comparison will determine the similarity measure between the considered image of brain tumor and that found in the database, this help to make the classification decision based on the detected type of brain tumor. The following subsections present more explanations about each stage of the proposed method:

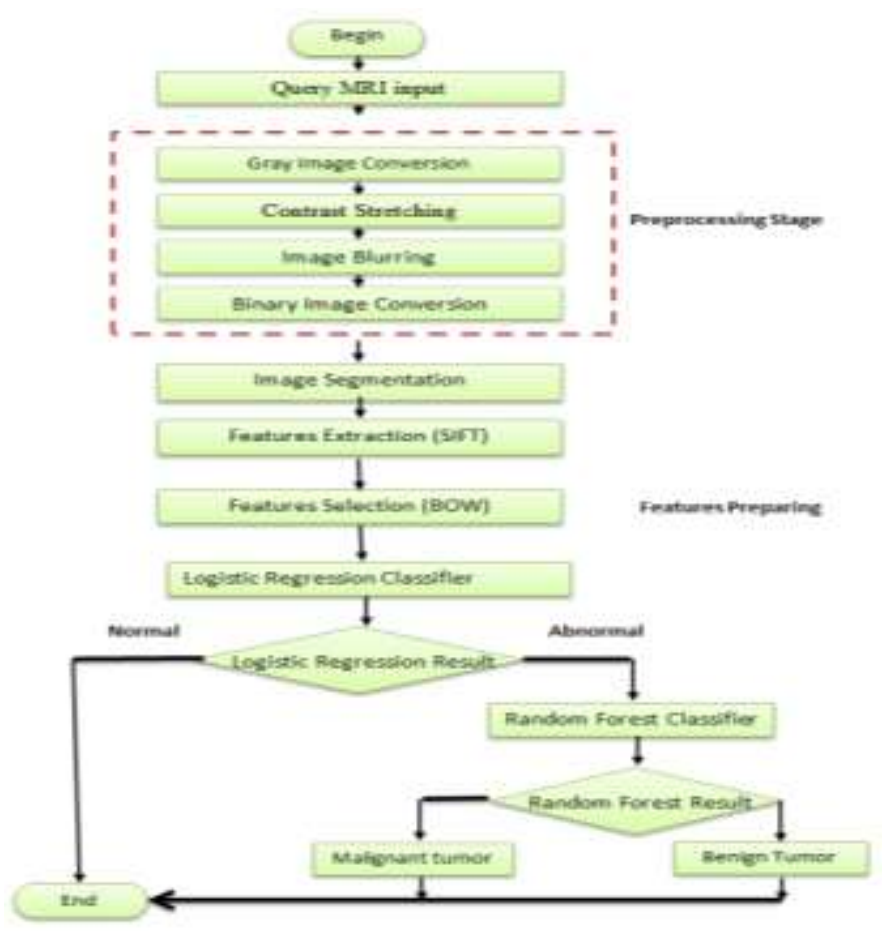

Figure 1. Block diagram of suggested image classification.

\subsection{Image Preprocessing}

Image preprocessing aims to prepare the input brain MRI for classification after applying four steps:

\subsubsection{Grey Image Conversion}

Images in general are provided in form of RedGreen-Blue (RGB) color space format, it is necessary for the processing system for converting the RGB images to gray ones. Converting RGB colored image into gray-scaled image is affecting some features of images, the major general impacted features are: shadow, sharpness, and 


\section{Al-Nahrain Journal of Science \\ ANJS, Vol.23 (2), June, 2020, pp. 67 - 74}

contrast. The most common way that used to do such conversion is depending on equaling the three-color components to result gray image. Whereas, the luminance band computed by equation (1) is look as gray and seems to be richer than that of gray image as shown in figure 2 .

$$
G=0.299 R+0.587 G+0.114 B \ldots \text { (1) }
$$
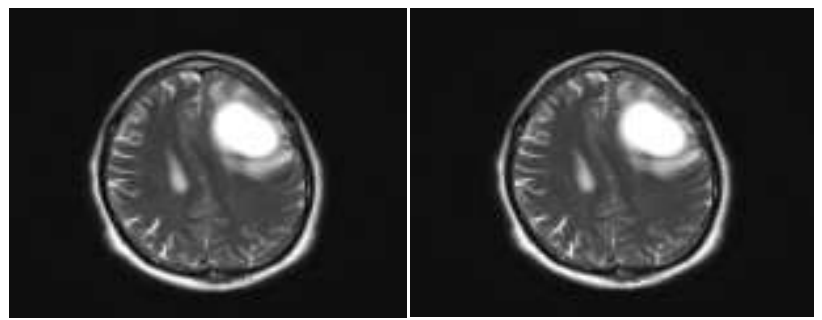

Figure 2. Gray scale MRI brain image (a) left: MRI sample image. (b) Right: Its gray image

\subsubsection{Contrast Stretching}

Contrast stretching shown in figure 3 involves the use of linear fitting model for stretching image's contrast. For a given image Ie, the measured minimum and maximum values of color values are Imin and Imax frequently, while intended maximum as well as minimum values regarding improved stretched image (S) are specified as Smin as well as Smax, after that, improved image (S) will be associated to spectral intensity image (I) through this linear relation:

$$
S=a \times I+b
$$

In which, a as well as b representing coefficients regarding linear fitting model between $\mathrm{S}$ as well as G. In the case of substituting Imin and Imax which are estimated from $\mathrm{G}$ image and recognized values regarding Smin and Smax in the eq.(2), the next 2 relations which contain only 2 unknowns a and b will be determined:

$$
\begin{aligned}
& S_{\text {max }}=a \times I_{\text {max }}+b \\
& S_{\text {min }}=a \times I_{\text {min }}+b
\end{aligned}
$$

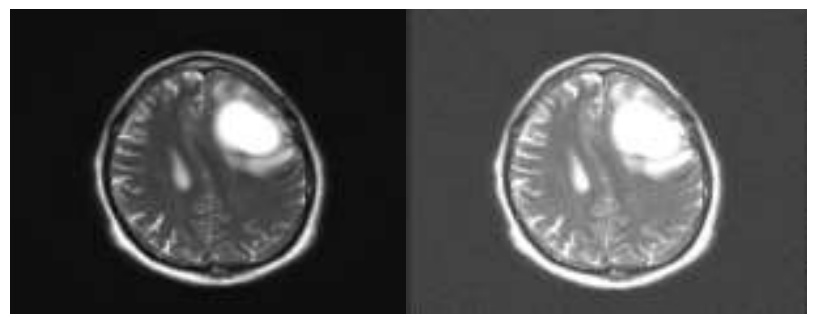

Figure 3. Contrast MRI brain image. (a) Left: MRI sample image. (b) Right: Its contrast image

\subsubsection{Image Blurring}

Image blurring shown in figure 4 is caused by using blurring function such as Gaussian that is generally applied for the reduction of image noise as well as the fine details and skull stripping. In this system, a 2D Gaussian is used on image with $9 \times 9$ mask

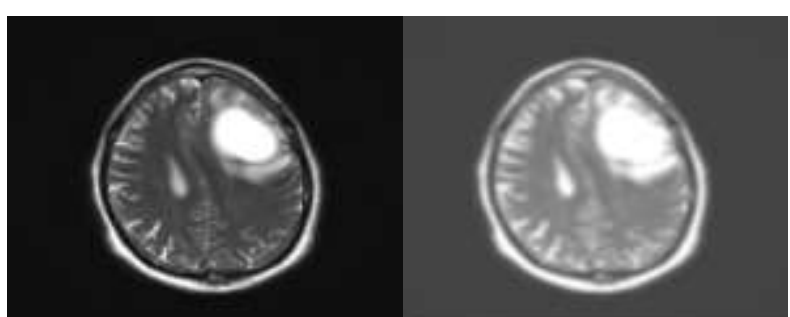

Figure 4. Blurring MRI brain image. (a) MRI sample image. (b) Its blurring image

\subsubsection{Binary Image Conversion}

Converting gray scale image to binary image is shown in figure 5, it requires a thresholding process which guarantee that only the region of interest (brain parts) will maintain inside image and any other information is discarded which led to have only two areas in the image the foreground which is represent the region of interest and the background which is black .In the system, thresholding value equal or greater than 240 and less than 255 .
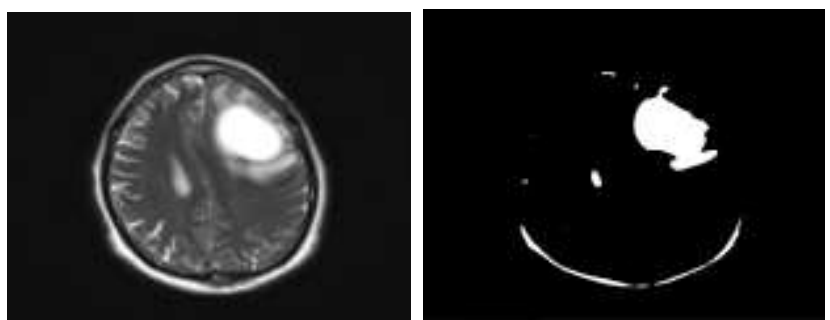

Figure 5. Binary MRI brain image. (a) MRI sample image. (b) Its binary image.

\subsection{Image Segmentation}

Image segmentation stage shown in figure 6 uses contour method to determine the ROI, which is the brain tumor that intended to extract its features in the following stage. This procedure is used to describe the curved boundary and counters to determine the boundary of brain tumor.

\subsection{Features Extraction}

SIFT is considered as a new class of algorithms that emerged to be used for object recognition, the basic idea is the use of local features matching by transforming the content of image into coordinates of local features which then invariant for 


\section{Al-Nahrain Journal of Science \\ ANJS, Vol.23 (2), June, 2020, pp. 67 - 74}

translation, rotation, and scaling. This method suggested by David Lowe for extracting features which are distinctive and invariant from image for recognizing different images of same object. According to SIFT algorithm, each input MRI contains a specific number of key points, each representing a vector feature of length 128 values. The number of features in each image depends on the image details found in that image.

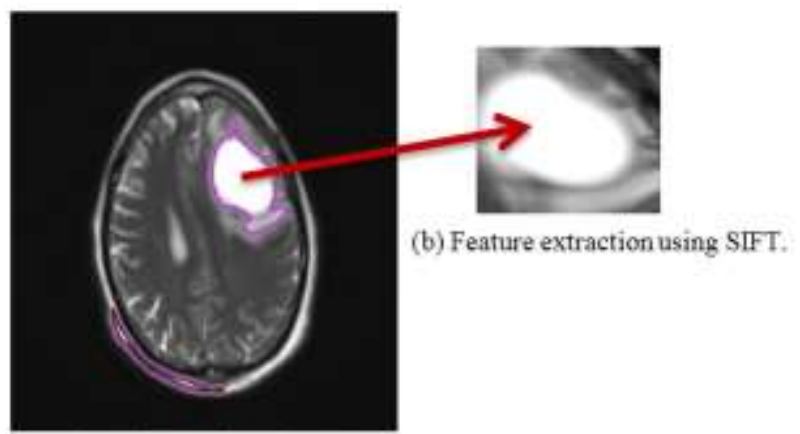

(a) Abnormal MRI image.

Figure 6. Feature extracted from abnormal MRI image

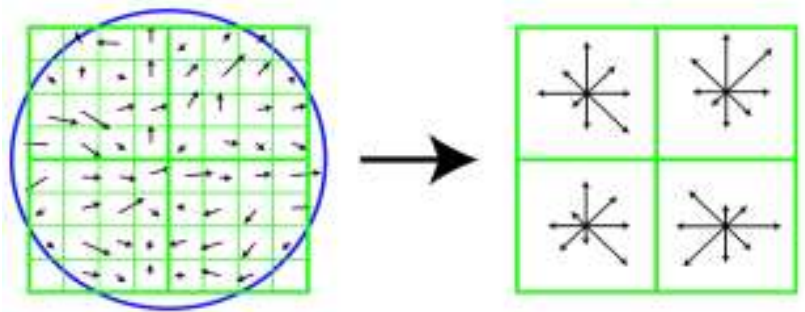

Figure 7. Computing the key-point descriptor.

\subsection{Classification Phase}

The classification phase is concerned with classifying the feature obtained from previous stages and detect if the segmented image is normal or abnormal tumor. Two levels of classifications are applied in the present system; the first level is to check if the tumor in the brain image is normal or abnormal, which is done using Logistic Regression Classification method. In case of normal situation, the system presents a message tells the user that the brain image is normal case, while when the handled case is abnormal then the image exported into later Random Forest classifier that check if the tumor detected in the brain image is Malignant (i.e., cancer) or it is Benign (i.e. Just a tumor).

\section{Results and Discussion}

In the practical test, different types of MRI are used in this procedure according to the requirement. Type of sequences used in MRI provided as an input in the preprocessing step like T1, T2 and FLAIR. The dataset is a collection of MRI were taken from the website (Radiopaedia), it is a free source of medical images for education process. Such images are considered to be contained two classes: normal and abnormal, in which the abnormal case may be benign or malignant. This dataset consist of 466 MRI images, figure 8 shows some samples of them.
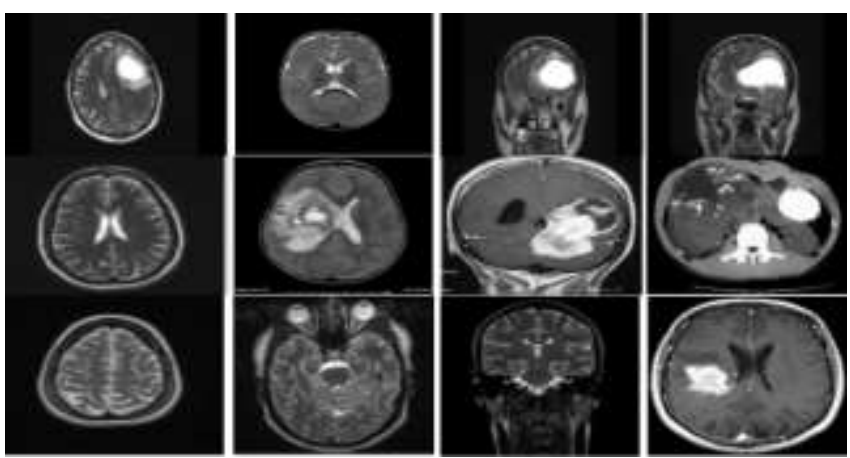

Figure 8. Samples of used MRI dataset.

The proposed system contains preprocessing the data to prepare the handled image by removing unwanted data and convert it into useful form that may help the classification phase which is responsible on decision making in present system. These images are needed to be preprocessed for minimizing the time needed for processing. figure 9 (a) shows the original MRI, while the result of applying the gray scale conversion is shown in (b), the result of blurring the gray scale image using $9 \times 9$ mask is shown in (c), then these resulted image were converted into binary image where only the tumor part maintain and any other details was discard automatically using thresholding as shown in (d), which is used as a mask to determine the ROI. the tool that determining the ROI is the contour, which is appear as a bounder that enclose the white part of the image that shown bounded as given in (e). As a result, figure 9 (f) shows the segmented part that represents infected region. 


\section{Al-Nahrain Journal of Science \\ ANJS, Vol.23 (2), June, 2020, pp. $67-74$}

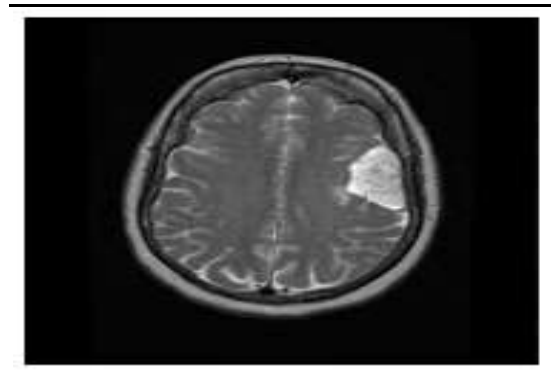

(a) MRI

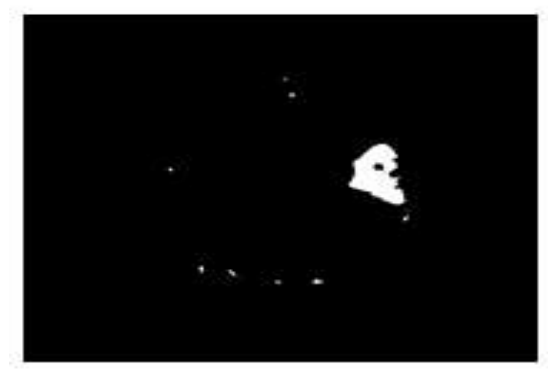

(d) Binary image

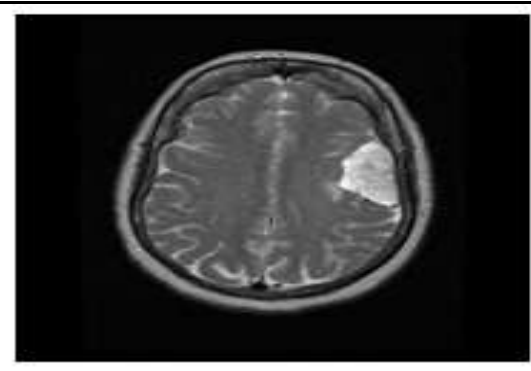

(b) Gray image.

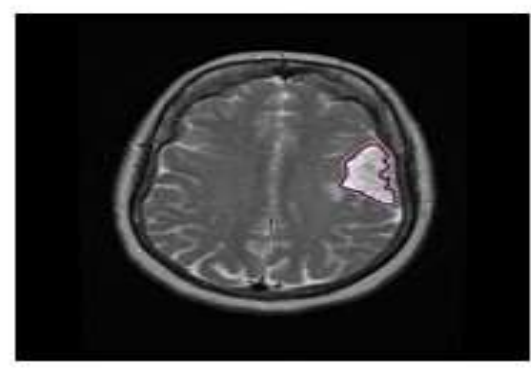

(e) Contoured Image.

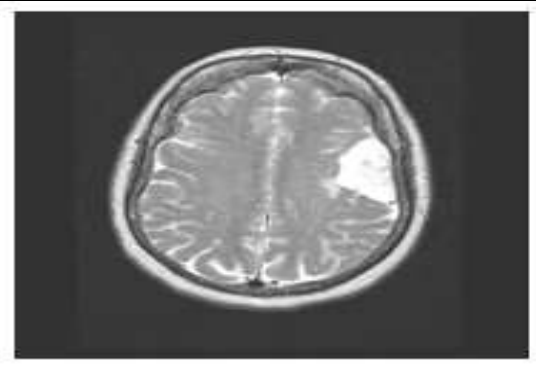

(c) Blurred image

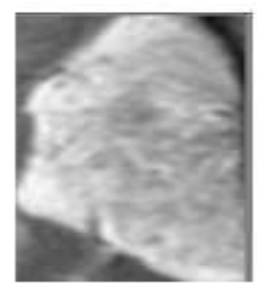

(f) Segmented image

Figure 9. Results of preprocessing stage including the adopted six steps applied on abnormal sample-1 from MRI dataset.

Figurer 10 shows the infected MRI image where only the ill part segmented and processed using SIFT. Figure 11 shows the feature extraction process using SIFT method. In figure 11 (a) showed the Different Of Gaussian DOG discrete extreme using SIFT, figure 11 (b) represents discrete extreme after Thresholding the DOG value using SIFT, figure 11 (c) shows candidate key points after refinement of 3D extreme position using SIFT, figure (d) Candidate key points after Thresholding interpolated DoG value SIFT, figure 11 (e) presents candidate key points after discarding those laying on SIFT edges, and figure 11 (f) shows final SIFT key points with assigned reference SIFT orientation.

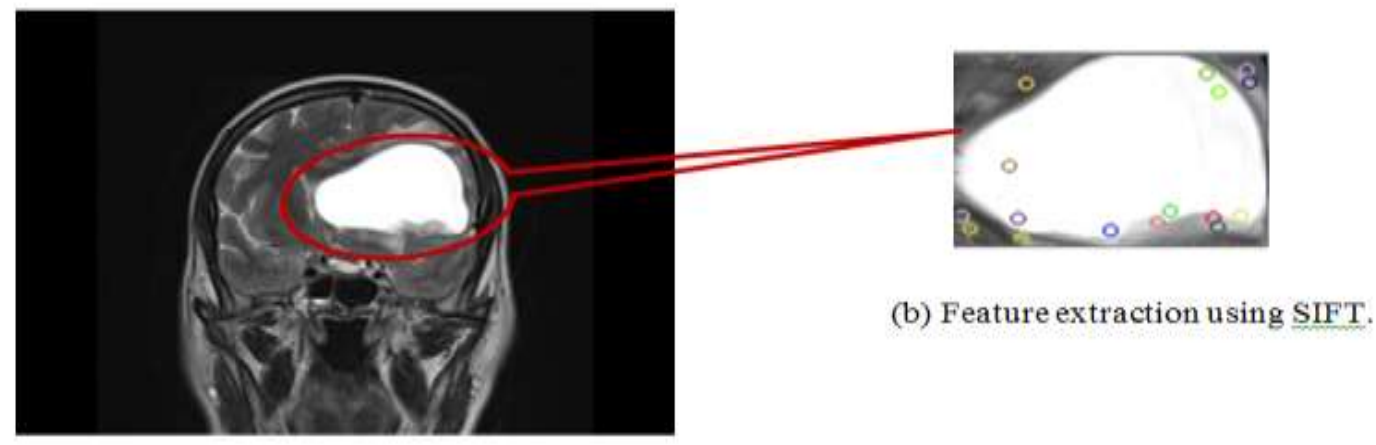

(a) Abnormal MRI image.

Figure 10. Feature extracted from abnormal MRI image. 


\section{Al-Nahrain Journal of Science}

ANJS, Vol.23 (2), June, 2020, pp. $67-74$

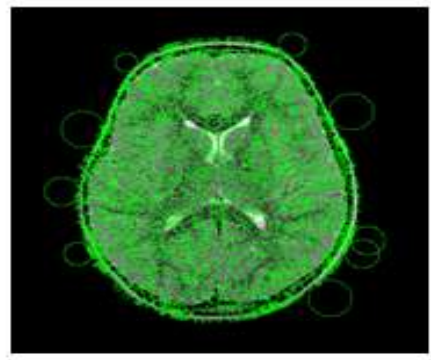

(a) DoG discrete extrema.

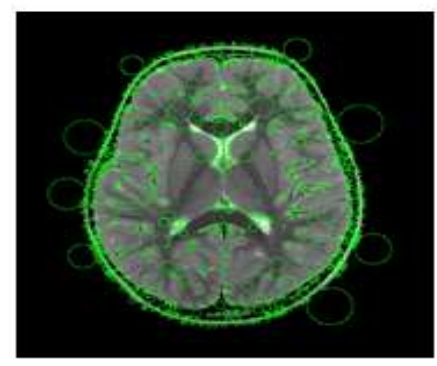

(d) Interpolated DoG

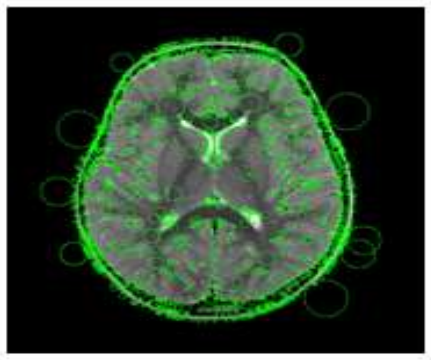

(b) Thresholding DoG.

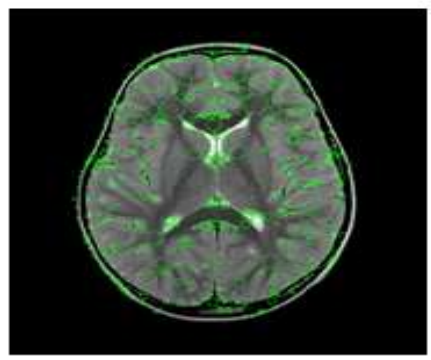

(e) Edges exclusion.

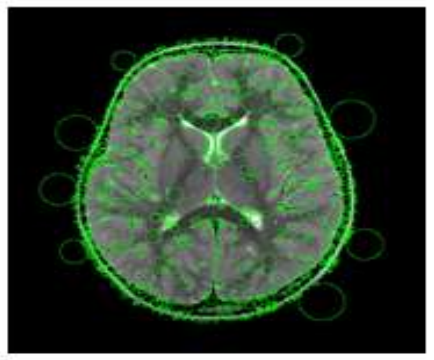

(c) Candidate key points.

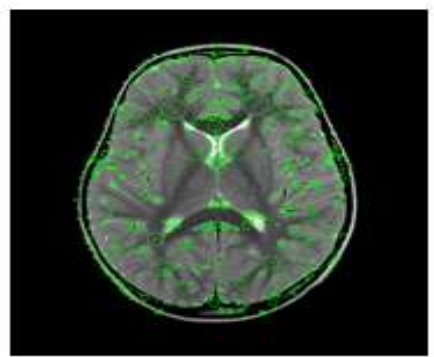

(f) Orientation assignment

Figure 11. Results of SIFT features extraction method including six steps applied on normal MRI image

128 features extracted from each key point in image and need to be reduced in order to keep only the strong features that can provide active classification and speed up the whole detection of tumor operation this is done in our system using BOW algorithm. Table (1) illustrates the true classification rate of the LR classifier that classifies the MRI into normal and abnormal case, which gave a classification percent of about $93.195 \%$. Whereas, the true classification rate of and $\mathrm{RF}$ classifier is given in Table (2), where the classification rate was about $99.996 \%$. It is noticeable that both classification scores are acceptable and nearly to that achieved by the two classifiers of the proposed method.

Table 1. LR classification Results.

\begin{tabular}{ll}
\hline Pointer & Resulted Percentage \\
\hline True Positive Rate & 93.1950277883840 \\
Precision & 93.3789451319558 \\
Recall & 93.1950277883840 \\
mean Absolute Error & 12.1510904976604 \\
root Mean Squared Error & 24.055014431062 \\
Total Number of Instances & 117855.0 \\
Correct & 109835.0 \\
Incorrect & 8020.0 \\
\hline
\end{tabular}

Table 2. RF classification results.

\begin{tabular}{ll}
\hline Pointer & Resulted Percentage \\
\hline True Positive Rate & 99.9966268636 \\
Precision & 99.9966270194 \\
Recall & 99.9966268636 \\
mean Absolute Error & 0.00168656818 \\
root Mean Squared Error & 0.29039354082 \\
Total Number of Instances & 29646.0 \\
Correct & 29645.0 \\
Incorrect & 1.0 \\
\hline
\end{tabular}

\section{Conclusions}

The present study is considered to be an effort of high importance for revealing the significance regarding image classification with regard to the field of bio-computing. MRI is an imaging technique which is useful for providing enough information for disease diagnosis and decision making. Utilizing preprocessing with the aid of blurring was useful to skull stripping and remove noise from images and converts it to binary image. SIFT descriptor applied on MRI was successful to describe the distribution of spectral details around the tumor in the brain image. SIFT descriptor is extract the features from images never the less of the direction or lightness or the size of an image. Cross validation improves the classification results since all the data set image participates in the classification process. Such systems cannot provide final answer for the type of tumor using only one 


\section{Al-Nahrain Journal of Science}

ANJS, Vol.23 (2), June, 2020, pp. $67-74$

classification method, so two algorithm is used to detect the tumor if it is normal or abnormal and the second one is to show the exact type of the abnormal tumor (cancer or not). The use of Logistic Regression or even linear regression was successful to achieve high classification percent to diagnosis the brain image into infected or not according to the selected features of the SIFT descriptor. The use of Random Forest or even random forest was also successful to provide high classification percent to diagnosis the tumor of the brain image into benign or malignant according to the selected features of the SIFT descriptor.

\section{References}

[1] Tahir; M., N.; "Classification and characterization of brain tumor MRI by using gray scaled segmentation and DNN, "International Joint Conference on Computer Vision Theory and Applications, 2018.

[2] Gopal, S. T.; "A Review on a Deep Learning Perspective in Brain Cancer Classification." International Joint Conference on Computer Vision Theory and Applications Cancers 11(1), 111, 2019.

[3] Abdulla, A. S.; Bushra, Q. A. A.; Mohammed, S. M.; "Classification of Al-Hammar Marshes Satellite Images in Iraq using Artificial Neural Network based on Coding Representation." Ind. J. Ecol. 45(4), 728-736, 2018.

[4] Dandu, J. R.; "Brain and pancreatic tumor segmentation using SRM and BPNN classification." Health and Technology, 1-9, 2019.

[5] Cheung, W.; Ghassan, H.; "SIFT in 3Dimensional Scale Invariant Feature Transform." IEEE Trans. Im. Proc. 18(9), 2021, 2012.

[6] Mohammed, S. M. A.; Saif, M. A.; "Satellite Image Classification using Multi Features Based Descriptors", Int. Res. J. Adv. Eng. Sci., 2018.
[7] Sachdeva, J.; "A package-SFERCB-"Segmentation, feature extraction, reduction and classification analysis by both SVM and ANN for brain tumors"." Applied soft computing 47, 151-167, 2016.

[8] Kamavisdar, P.; Sonam, S.; Sonu, A.; "A survey on image classification approaches and techniques." Int. J. Adv. Res. Comp. Comm. Eng. 2(1), 10051009, 2013.

[9] O'Tool, A. J.; "Theoretical, statistical, and practical perspectives on pattern-based classification approaches to the analysis of functional neuroimaging data." J. Cog. Neuro. 19(11), 17351752, 2007.

[10] Amulya, C.; Prathibha, G.; "MRI Brain Tumor Classification Using SURF and SIFT Features." Int. J. Modern Tren. Sci. Technol. 2(7) 123-127, 2016.

[11] Alfonse, M.; Abdel, B. M. S.; "An automatic classification of brain tumors through MRI using support vector machine." Egy. Comp. Sci. J. 40(3), 2016.

[12] Gurusamy, R.; Vijayan, S.; "A machine learning approach for MRI brain tumor classification." Computers, Materials and Continua 53(2), 91-108, 2017.

[13] Bahadure, N. B.; Arun, K. R.; Har, P. T.; "Image analysis for MRI based brain tumor detection and feature extraction using biologically inspired BWT and SVM." Int. J. Biomed. Im., 2017.

[14] Bahadure, N. B.; Arun, K. R.; Har, P. T. ; "Comparative approach of MRI-based brain tumor segmentation and classification using genetic algorithm.", J. Digit. Imag. 31(4), 477-489, 2018.

[15] Dandu, J. R.; "Brain and pancreatic tumor segmentation using SRM and BPNN classification.", Health and Technology 1, 2019.

[16] Polepaka, S.; Ch, S. R.; Chandra, M.; "A Brain Tumor: Localization Using Bounding Box and Classification Using SVM.", Innov. Electro. Commun. Eng., 61-70, 2019. 